\section{FEBRUARY 2009}

\section{EACTS Course Cardiac and}

Thoracic Robotic Surgery

Course: February 4-7, 2009, Strasbourg, France. For more information, contact: EACTS Executive Secretariat, 3 Park Street, Windsor. Berkshire SL4 1LU, UK; (telephone: +44 1753 832166; fax: +44 1753 620407; E-mail: info@eacts.co.uk; Website: http://courses.eacts.org/sections/CT/ RobSurg/index.html).

\section{Introduction to Cardiothoracic Surgery}

Meeting: February 6, 2009, Adamson Centre, Edinburgh, Scotland. For more information, contact: Laura McQuade, Course Co-ordinator, The Royal College of Surgeons of Edinburgh, The Adamson Centre, 3 Hill Place, Edinburgh, EH8 9DS, UK (Telephone: +44 0(0) 131668 9238; E-mail: 1.mcquade@rcsed.ac.uk).

\section{The 6th Annual Atlanta Lung Cancer Symposium}

Symposium: February 7, 2009 at Omni Hotel, Atlanta, Georgia. For more information, contact: Jackie Falconer, Educational Concepts Group, 1300 Parkwood Circle, Suite 325, Atlanta, GA 30339 (Telephone: 1- 770933-1696; Fax: 1- 770- 933-1692; E-mail: jfalconer@educationalconcepts. net).

Florida Valve 2009 - Valvular Heart Disease for the Surgeon and Cardiologist: New Strategies that Unify Practice

Meeting: February 27, 2009- 1 March 1, 2009, Renaissance Vinoy Resort, St. Petersburg, Florida. For

\footnotetext{
$\overline{\text { Announcements }}$ of major meetings and other significant activities must be received at least 8 weeks before the desired month of publication. Information will be limited to title of meeting, date, place, and an address to obtain further information. Send announcements to Ryan Walther, Managing Editor, The Journal of Thoracic and Cardiovascular Surgery, 900 Cummings Center, Suite 221-U, Beverly, MA 01915 (E-mail: rwalther@prri.com).
}

more information, contact: Tampa Bay Heart Foundation, Inc. (Telephone: 1- 727- 522-9379; Fax: 1- 727- 5229386; E-mail: info@TBHFoundation. org).

\section{MARCH 2009}

\section{Re-Evolution Summit: Minimal Access Cardiac Surgery}

Meeting: March 5-7, 2009, Hotel ZaZa, Houston, Texas. For more information, contact: Veronica Lopez, Office of Continuing Medical Education, 6565 Fannin, MGJ9-004, Houston, TX 77030 (Telephone: 1- 713- 4414971; Fax: 1- 713- 441-0589; E-mail: vlopez2@tmhs.org).

The 17th Annual Meeting of the Asian Society for Cardiovascular and Thoracic Surgery (ASCVTS) and the 2nd AATS/ ASCVTS postgraduate course

Meeting and course: March 5-8, 2009, Taipei International Convention Center, Taipei Taiwan. For more information, contact: Elite Professional Conference Organizer, Ms. Cheryl LIN, 10F-2, No. 51, Sungjiang Road; Taipei 104, Taiwan (Telephone: $+886-$ 2-2504-4338 Ext. 16; Fax: +886-22504-4362; E-mail: ascvts2009@elitep co.com.tw).

\section{$24^{\text {th }}$ International Interventional Cardiology 2009 Symposium}

Meeting: March 8-13, 2009, Silvertree Hotel, Snowmass Village, Colorado. Abstract submission deadline: January 23, 2009. For more information, contact: Laurel Steigerwald, 2333 State Street, Suite 203, Carlsbad, California 92008 (Telephone: 1- 760- 7202263; Fax: 1 -760- 720-6263; E-mail: education@promedicacme.com).

The Annual Meeting of the General Thoracic Surgical Club Meeting: March 12-15, 2009, Don Cesar Beach Resort, St. Petersburg, Florida. For more information, contact: Bonnie Lemmerman or Carla MacLean, General Thoracic Surgical
Club, Mayo Clinic, 200 First Street SW, Rochester, MN 55905 (Telephone: +1 -507-538-4969; Fax: + 1 507284-0058; E-mail: blemmerman@mayo. edu or cmaclean@mayo.edu)

European School for CardioThoracic Surgery Cardiac Course Level A

Meeting: March 16-21, 2009, Bergamo, Italy. For more information, contact: EACTS Executive Secretariat, 3 Park St., Windsor, Berkshire S14 1LU, UK (Telephone: +44-1753832-166; Fax: +44-1753-620407; E-mail: info@eacts.co.uk).

Society for Cardiothoracic Surgery in Great Britain and Ireland 2009 Annual Meeting and Cardiothoracic Forum

Meeting: March 22-24, 2009, Bournemouth International Center, Bournemouth, UK. Further details and abstract submission online at www.scts.org from September 1st 2008. Contact sctsadmin@scts.org

The Eighth International Symposium on Sympathetic Surgery (ISSS): Approaching Clinical Care With Clinical Science and VATS 2009: The Seventh International Symposium on Video-Assisted Thoracic Surgery

Combined Meeting: March 25-29, 2009, Sheraton New York Hotel \& Towers, New York, New York. For more information, contact: Melanie Heath, Associate Program Manager, The CBCE (The Center for Biomedical Continuing Education) (Telephone: 1- 972- 692-2051; Fax: 1- 214- 2600408; E-mail: mheath@thecbce.com).

\section{APRIL 2009}

Functional Ischaemic Mitral Regurgitation (FIMR)

Meeting: April 3, 2009, National Heart and Lung Institute, London United Kingdom. For more information, contact: Karina Dixon, National 
Heart and Lung Institute, Dovehouse Street, Imperial College, London, SW3 6LY UK (Telephone: +44- 20- 73518172; Fax: +44- 20- 7351- 8246; E-mail: academicevents.nhli@imperial. ac.uk).

\section{Houston Aortic Symposium: Frontiers in Cardiovascular Diseases - The Second in the Series}

Meeting: April 3-5, 2009, The Westin Oaks Hotel, Houston Texas. Abstract submission deadline: February 20, 2009. For more information, contact: Michelle Gherardi, 2333 State Street, Suite 203(Telephone: +1- 760720-2263; Fax: +1- 760- 720-6263; Email:mgherardi@promedicacme.com).

\section{Minimally Invasive Cardiac Surgery}

Meeting: April 17-18, 2009, Sheraton Nicolaus Hotel, Bari, Italy. Abstract submission deadline: January 31, 2009. For more information, contact: Giuseppe Straziota, Department of Cardiovascular and Thoracic Surgery, Anthea Hospital (Telephone: +39- 08- 085644570; Fax: +39- 08- 0- 85644620; E-mail: info@minimally.it).

\section{European School for Cardio- Thoracic Surgery Cardiac Course Level A}

Meeting: April 20-25, 2009, Bergamo, Italy. For more information, contact: EACTS Executive Secretariat, 3 Park St., Windsor, Berkshire S14 1LU, UK (Telephone: +44-1753832-166; Fax: +44-1753-620407; E-mail: info@eacts.co.uk).

\section{The International Society for}

\section{Heart and Lung Transplantation} 29th Annual Meeting

Meeting: April 22-25, 2009, Paris France. For more information, contact: International Society for Heart \& Lung Transplantation, 14673 Midway Road, Suite 200, Addison, Texas 75001 (Telephone: 1- 972- 490-9495; Fax: 1- 972 490-9499; E-mail: ishlt@ishlt.org).
The 6th Vienna Interdisciplinary Symposium on Aortic Repair (VISAR)

Symposium: April 22-24, 2009, Congress Venue, Vienna Austria. Abstract submission deadline: February 28, 2009. For more information, contact: Congress Secretariat: E\&E PCO (Telephone: +43- 1- 867- 49- 44; Fax: +43- 1- 867-49-44-9; E-mail: office@ ee-pco.com).

\section{The 58th International Congress of the European Society of Cardiovascular Surgery (ESCVS)}

Congress: April 30 - May 2, 2009 in Warsaw, Poland. Notification of abstract acceptance: January 15, 2009. Low fee pre-registration: March 1, 2009. Scientific Secretariat: Prof. Claudio Muneretto (munerett@med.unibs. it). Organising Secretariat, Meet and Work srl, contact information: Mario Sbalchiero; Telephone +39- $049-860$ 1818; FAX +39- 049- 860- 2389; E-mail: meet@meetandwork.com).

\section{MAY 2009}

The 58th International Congress of the European Society for Cardiovascular Surgery Congress: May 1-4, 2009, Warsaw, Poland. For more information, contact: ESCVS General Secretariat, Prof. Claudio Muneretto, ESCVS Secretary General, UDA Cardiochirurgia, Spedali Civili P.le Spedali Civili 1, 25123 Brescia, Italy; (telephone: +39 030 3996401; fax: +39 030 3996096; E-mail: escvs.secretariat@gmail.com; Website: http://www.escvs.org/).

\section{The 8th International}

\section{Symposium on Endovascular}

Therapeutics

Symposium: May 7-9, 2009, Hotel Hilton, Barcelona, Spain. Abstract submission deadline: February 28, 2009. For more information, contact: Atlanta Unicongress Calvet 55 (Telephone: +34- 902- 109- 853; Fax: +34-
902- 109- 855; E-mail: site2009@ unicongress.org).

Eighth International Symposium on Redo Cardiac Surgery in Adults

Symposium: May 8-9, 2009 in Boston, Massachusetts. For more information, contact: University of Pittsburgh Medical Center, Division of Cardiac Surgery The Heart, Lung, and Esophageal Surgery Institute, Shadyside Medical Building, Suite 7065200 Centre Avenue Pittsburgh, PA 15232 (Telephone: 1- 412- 6233140; Fax: 1- 412- 623-2822; E-mail: slomkasm@upmc.edu).

The 55th Annual Conference of the Indian Association of Cardiovascular \& Thoracic Surgeons (CT CON 2009)

Conference: May 14-17, 2009, Srinagar, Kashmir, India. For more information, contact: Conference Secretariat, Prof. AG Ahangar, Head of Department of Cardiovascular \& Thoracic Surgery. Sher-I-Kashmir Institute of Medical Sciences, Soura, Srinagar, India; Tel: +91- 194- 2400348; E-mail: iacts2009@yahoo.com)

\section{European School for Cardio- Thoracic Surgery Cardiac Course Level B}

Meeting: May 18-23, 2009 Bergamo, Italy. For more information, contact: EACTS Executive Secretariat, 3 Park St., Windsor, Berkshire S14 1LU, UK (Telephone: +44-1753832-166; Fax: +44-1753-620407; E-mail: info@eacts.co.uk).

\section{Postgraduate Graduate Course in General Thoracic Surgery \\ Course: May 21-22, 2009, Boston, Massachusetts. For more information: Call: (617) 384-8600, 10:00 am-4:00 pm Eastern Time, Monday-Friday; Fax: (617) 384-8686; E-mail: hms-cme@ hms.harvard.edu.}




\section{The XIV Congress of the Catalan Society for Cardiac Surgery}

Congress: May 27-30, 2009, Hotel Avenida Palace, Barcelona Spain. Abstract submission deadline: March 15, 2009. For more information, contact: Nuria Bosch - Oldisseny S.L., Comte (Telephone: +34- 934- 510- 792; Fax: +34- 934- 511- 736; E-mail: nbosch@ oldisseny.com).

\section{5th International Conference on \\ Pediatric Mechanical \\ Circulatory Support Systems \& \\ Pediatric Cardiopulmonary \\ Perfusion}

Conference: May 27-30, 2009, Dallas, Texas. For more information: pedsabstracts@hmc.psu.edu.Website: http://www.hmc.psu.edu/childrens/ pedscpb/

\section{ASAIO - IFAO Annual}

Conference in conjunction with

the 5th Annual Pediatric

Mechanical Circulatory Support

Systems \& Pediatric

Cardiopulmonary Perfusion

Conference: May28-30, 2009, Hilton Anatole Hotel, Dallas, Texas. Abstract submission deadline: January 15, 2009. For more information, contact: ASAIO Inc., 980 North Federal Highway, Suite 212, Boca Raton, Florida 33432-2711 (Telephone: 1561- 391-8589; Fax: 1 -561-368-9153; E-mail: info@asaio.com)

\section{3rd Ukrainian-Polish Congress of Cardiovascular Surgery}

Meeting: May 28-29, 2009, Kyiv, Ukraine. For more information, contact: M.M. Amosov National Institute of Cardiovascular Surgery of the Academy of Medical Sciences of Ukraine, 6 M.Amosov Street, MSP 03680 (Telephone:: +38 (0)44- 27543-22; Fax: +38 (0)44 -275-43-22;

E-mail: info@amosovinstitute.org.ua).

17th European Conference on General Thoracic Surgery

Conference: May 31 - June 3, 2009, Krakow, Poland. Abstract Sub- mission Deadline: February 1, 2009. For more information, contact: Mondial Congress and Events, Mondial GrmbH \& Co., Operngasse 20b, 1040 Vienna, Austria; (telephone: +431 588040 ; fax +431588040185 ; E-mail: ests2009@mondial-congress. com; Website: http://www.estsmeetings. org/2009/).

\section{JUNE 2009}

Fifth Biennial Meeting of The Society for Heart Valve Disease

Meeting: June 2009, Ritz Carlton Hotel, Berlin Germany. For information, contact: SHVD Executive Secretariat, Heart Science Centre, Harefield Hospital, Harefield Middlesex, UB9 $6 J H$, UK (telephone: +44 (0)1895828977; fax: +44 (0)1895-828902; E-mail: secretariat@shvd.org; Website: www.shvd.org).

The 2009 International Society for Minimally Invasive Cardiothoracic Surgery (ISMICS) 12th Annual Scientific Meeting

Meeting: June 3-6, 2009, Westin St. Francis in San Francisco, California. For more information, contact: EJ Weldon, 900 Cummings Center, Suite 221-U, Beverly, MA (Telephone: +1 978-927-8330; E-mail: ISMICS@prri.com).

\section{Practices in Cardiac Surgery and} Extracorporeal Technologies The New Orleans Conference

Conference: June 10-13, 2009, Westin Canal Place Hotel, New Orleans, Louisiana. For more information, contact: Joseph Basha, LP, CCP, CEO: Perfusion International, LLC, Program Director: The New Orleans Conference (Telephone: 1318- 623-0890; E-mail: JosephBasha@PerfusionInternational.com)

European School for CardioThoracic Surgery Cardiac Course Level B

Meeting: June 15-20, 2009, Bergamo, Italy. For more information, contact: EACTS Executive Secretariat, 3 Park St., Windsor, Berkshire S14 1LU, UK (Telephone: +44-1753832-166; Fax: +44-1753-620407; E-mail: info@eacts.co.uk).

The 5th World Congress of Paediatric Cardiology and Cardiac Surgery

Congress: June 22-26, 2009, Cairns Convention Centre, Cairns, Queensland Australia. For more information, contact: ICMS Pty Ltd (Telephone: +613- 9682- 0244; Email: pccs2009@ icms.com.au)

The Western Thoracic Surgical Association (WTSA) 35th

Annual Meeting

Meeting: June 24-29, 2009, Fairmont Banff Springs Hotel, Banff, AB Canada. For more information, contact: April Conti; 900 Cummings Center; Suite 221-U, Beverly, MA 01915 (Telephone: +1 978-927-8330; FAX: +1978-524-8890; Email: wtsa@prri. com).

\section{JULY 2009}

The 13th World Conference on Lung Cancer (WCLC 2009)

Conference: July 31 - August 4, 2009. San Francisco, Califormia, USA. Abstract Submission Deadline: March 13, 2009. For more information, contact: Khara Robertson, Conference Manager, (telephone: 1604-681-2153, e-mail atwclc2009@ meet-ics.com; Website: http://www. 2009 worldlungcancer.org/).

Event Name: $6^{\text {th }}$ Annual ExtraCorporeal Membrane Oxygenation course

Date: March 9 - 11, 2009

Conference Center (venue): King Faisal Specialist Hospital \& Research Center

City: Jeddah

Country: Saudi Arabia

Address: MBC-J 16, P.O. Box, Jeddah 21499, Saudi Arabia

Abstract Due Date: Not applicable 
Web Site: www.kfshrcj.org (click on more events)

Contacts:

1- Mr.Faiz Al-Malki (ECMO coordinator)

Phone: 9662-667-7777 ext 2166

Fax: 9662-663-9581

e-mail: faizwinner@gmail.com

2- Ms. Sonnet Liwanag (Hospital assistant)

Phone: 9662-667-7777 ext 5234

Fax: 9662-663-9581

e-mail: sliwanag@kfshrc.edu.sa

\section{SEPTEMBER 2009}

European School for Cardio-

Thoracic Surgery Cardiac

Course Level C

Meeting: September 21-26, 2009,

Bergamo, Italy. For more information, contact: EACTS Executive Secretariat, 3 Park St., Windsor, Berkshire S14 1LU, UK (Telephone: +44-1753-832166; Fax: +44-1753-620407; E-mail: info@eacts.co.uk).

\section{OCTOBER 2009}

Venice 2009 Arrhythmias - 11th International Workshop on Cardiac Arrhythmias

Workshop: October 4-7, 2009, Fondazione Vini, Venice, Italy. Abstract submission deadline: April 7, 2009. For more information, contact: Rita Reggiani - Project Leader, Rimini, Italy (Telephone: +39- 0541- 305822305811; Fax: +39 -0541-305842; E-mail: infor@venicearrhythmias.org)
The 23 $^{\text {rd }}$ European Association for Cardio-Thoracic Surgery (EACTS) Annual Meeting

Meeting: October 17-21, 2009, Austria Centre, Vienna, Austria. Abstract submission deadline: April 1, 2009. For more information, contact: EACTS Executive Secretariat, 3 Park St., Windsor, Berkshire SI4 ILU, UK (Telephone: +44-1753-832-166; Fax: +44-1753620407;E-mail:info@eacts.co.uk).

Association of Thoracic and Cardiovascular Surgeons of Asia (ATCSA) 19th Biennial Congress 2009

Congress: October 25-28, 2009, Sheraton Grande Walkerhill Hotel, Seou, Republic of 1 Korea. Abstract submission deadline: June 1, 2009. For more information, contact: Ms. Hyo-Shin Nam, InSession International Convention Services, Inc (Phone: $+82-$ 2 -3471 8555; Fax: +82- 2- 521- 8683; E-mail: atcsa2009@insession.co.kr)

Vanderbilt Valve Symposium: 21st Century Techniques for Complex Valve Surgery

Symposium: October 29-30, 2009, Nashville Marriott Hotel, Nashville, Tennesse. For more information, contact: Joan Lorber, Vanderbilt Heart, Department of Surgery, 1215 21st Avenue South, 5209 MCE, Nashville, TN 37232-8802(Telephone:1- 615- 3437363; E-mail: joan.lorber@vanderbilt. edu).

\section{NOVEMBER 2009}

\section{European School for Cardio- \\ Thoracic Surgery Cardiac \\ Course Level C}

Meeting: November 9-14, 2009, Bergamo, Italy. For more information, contact: EACTS Executive Secretariat, 3 Park St., Windsor, Berkshire S14 1LU, UK (Telephone: +44-1753832-166; Fax: +44-1753-620407; E-mail: info@eacts.co.uk).

The 5th International Meeting of the Onassis Cardiac Surgery Center: Current Trends in Cardiac Surgery and Cardiology

Meeting: November 12-14 2009, Athens Hilton Hotel, Athens, Greece. For more information, contact: Liana Iliopoulou, Triaena Tours \& Congress, 206 Sygrou Avenue, 17672 Athens (Kallithea) Greece (Telephone: +30 210- 7499353; Fax: +30- 2107705752; E-mail: lianae@triaenatours. gr).

The International Joint Meeting on Thoracic Surgery

Meeting: November 25-27, 2009, Auditorio Fomento de Trabajo Barcelona, Barcelona Spain. Abstract submission deadline: May 15, 2009. For more information, contact: Oriol Seto, ACTO SERVEIS, C/ Bonaire, 7; 08301 Mataro, Barcelona, Spain (Telephone: +34 -937- 552- 382; Fax: +34 -937- 552- 383; E-mail: thoracic.surgery@actoserveis.com). 